The use of specific $\mathrm{H}_{1}$ and $\mathrm{H}_{2}$ antagonists to analyse brain histamine action has been carried also into another phylum. Recently, Nahorski, Rogers and Smith (Br. J. Pharmac, 52, $121 \mathrm{P}$; 1974) in experiments carried out in the cerebral hemispheres of the chick, in vivo and in vitro, found that $\mathrm{H}_{2}$ receptor antagonists blocked histamine-induced cyclic AMP formation, whereas an $\mathbf{H}_{1}$ antagonist was ineffective. Thus, the chick may differ from the guinea pig in that histamine receptors on its cortical adenyl cyclase may be largely of $\mathrm{H}_{2}$ type, whereas in the guinea pig both types seem to be represented.

\section{Glassy crystals}

from Robert W. Cahn

IN his stinging attack on Plato's political philosophy, Karl Popper (The Open Society and Its Enemies, chapter 3; Routledge, 1945) castigates Plato's "methodological essentialism", the delusion that to seek, by defining it, the essence of a thing can be a valid way of arriving at knowledge about the external world-including the world of social relations. Popper assures us that a true scientist always steers clear of questions which tempt him to definition-mongering, such as "What is an atom?" I am not at all sure that this is universally true: a passion for definitions sometimes provokes a scientist into a programme of experimentation which he might otherwise never have undertaken. The quest for the word provokes the deed.

These reflections are aroused by an unusual paper on the glassy state (Suga and Seki, J. Non-Cryst. Solids, 16, 171; 1974). The authors, chemists at Osaka University, consider various ways of making non-crystalline solids other than by supercooling of a liquid, and ask whether it is plausible to call such solids "glasses". By itself, such a question deserves all the obloquy that Popper would direct upon it; but Suga and Seki went on to point out that the existence of a glass transition temperature is the crucial characteristic that has gradually come to be the defining criterion for glassiness. Thereupon they were moved to undertake a systematic study of glass transitions in a number of pure compounds nudged into noncrystalline states by cooling the melt, by condensing the vapour or by other more unorthodox means. In the course of these studies, performed in specially designed precision calorimeters (DTA apparatus), the authors discovered a number of anomalies: one compound might show more than one glass transition, and the concept of glassiness therefore required subdivision. It is rather as though St Thomas Aquinas had conducted microscopic observations on the creatures cavorting on his needle-point and decided that it was necessary to distinguish archangels from mere angels.

Apart from a number of simple organic compounds such as isopentane, methanol, isopropylbenzene and cyclohexanol, the authors also examined a number of inorganic and organic high polymers, some inorganic materials $\left(\mathrm{H}_{2} \mathrm{O}, \mathrm{SnCl}_{2} .2 \mathrm{H}_{2} \mathrm{O}\right)$ and a range of compounds that form nematic or smectic (liquid crystal) phases. Thirty-eight materials were studied.

With regard to many of the simple compounds, it turned out that ordinary glass transitions, in the form of anomalies in the specific heat, turned up irrespective of whether the non-crystalline forms were prepared by supercooling a liquid, by condensation from the vapour or by rapid precipitation from solution. This answered the original question.

But three kinds of anomalous glass transitions were also found in some materials in addition to the ordinary transitions. Some compounds (for example, cyclohexanol, 2,3-dimethylbutane) have been known for some time to possess an intermediate structure at temperatures between full crystallinity and full liquidity, the so-called 'plastic crystal' state. The molecules are positionally ordered but orientationally disordered and mobile. This makes the molecules dynamically pseudo-spherical; because of this, they crystallise in face-centred cubic or body-centred cubic structures, which are unusually prone to plastic deformation: hence the name. Suga and Seki found that by rapid cooling they were able to freeze in this anomalous state, which they christened glassy crystal; on gradual reheating, the metastable glassy crystals passed through a 'glass transition' at which the metastable form transformed irreversibly into a fully ordered crystalline polymorph.

Another form of glassy crystal was found by supercooling of water, $\mathrm{SnCl}_{2} 2 \mathrm{H}_{2} \mathrm{O}$ or $\mathrm{SnCl}_{2} 2 \mathrm{D} . \mathrm{O}$. Here the partial disorder was attributed to a freezing-in of the irregular positions of protons or deuterons; in this connection, the different transition temperatures for the last two compounds were of particular interest. There is a particularly full historical discussion of glassy ice-which, incidentally, has nothing whatever to do with the notorious polywater of a few years ago!

Finally, Suga and Seki examined a number of liquid crystals, in which there is orientational order, accompanied by positional disorder of molecules. It proved possible to 'freeze' this state by rapid cooling. The steady variation with temperature of the degree of molecular alignment, in the 'swarms' which are the liquid-crystal equivalents of ferromagnetic domains, is thereby inhibited. When the glassy liquid crystal, as the authors call this state, is heated through a transition, the molecules regain their labile ability to vary their degree of alignment.

Two compounds-cyclohexene and ethanol-were found to possess two anomalous transitions each as well as a normal transition. The nature of the two anomalous glassy crystalline states is not yet known.

The authors document their findings not only by locating glass transitions but also by computing the variation of entropy with temperature: from these plots they are able to make further deductions about the nature of the change they have observed.

The concept of a glassy crystal was sometime a paradox, but now the time gives it proof. Suga and Seki's paper is an impressive achievement which should stimulate many structural studies to confirm and extend their bold but sometimes tentative interpretations. Such studies may also have a bearing on topochemical reactions (see the detailed and very instructive review by J. M. Thomas, Phil. Trans. R. Soc., 277, 251; 1974). Topochemistry is concerned with the nature and kinetics of chemical reactions, including polymerisation reactions, between adjacent molecules in crystals, and such processes are bound to be modified if the state of positional and orientational order of molecules can be varied and then frozen into a particular pattern. Perhaps compounds can now be found in which internal reaction mechanisms will change at glass transition temperatures. If this proves to be so, we shall need a new definition to denote the process: vitrochemistry, perhaps?

\section{Behaviour in Europe}

\section{from a Correspondent}

THE first European Neurosciences meeting will be held in Munich next September (for details see the advertisement on page vii). This meeting represents the culmination of seven years' effort to promote collaboration between workers in different European countries and in different disciplines within the field of brain and behaviour research. The meeting is organised by the European Training Programme in Brain and Behaviour Research (ETP), a body whose history is of somc interest.

In 1968, the Organisation for Economic Cooperation and Development inaugurated studies of three scientific fields: the criteria for selecting fields were that they should be multi-disciplinary, have a fast growth rate, have insufficient recognition, and 